\title{
Genetic basis of channelopathies and cardiomyopathies in Hong Kong Chinese patients: a 10-year regional laboratory experience
}

\author{
Chloe M Mak *, Sammy PL Chen, NS Mok, WK Siu, Hencher HC Lee, CK Ching, PT Tsui, NC Fong, \\ YP Yuen, WT Poon, CY Law, YK Chong, YW Chan, TC Yung, Katherine YY Fan, CW Lam
}

\begin{abstract}
A B S T R A C T
Introduction: Hereditary channelopathies and cardiomyopathies are potentially lethal and are clinically and genetically heterogeneous, involving at least 90 genes. Genetic testing can provide an accurate diagnosis, guide treatment, and enable cascade screening. The genetic basis among the Hong Kong Chinese population is largely unknown. We aimed to report on 28 unrelated patients with positive genetic findings detected from January 2006 to December 2015.
\end{abstract}

Methods: Sanger sequencing was performed for 28 unrelated patients with a clinical diagnosis of channelopathies or cardiomyopathies, testing for the following genes: KCNQ1, KCNH2, KCNE1, $K C N E 2$, and $S C N 5 A$, for long QT syndrome; $S C N 5 A$ for Brugada syndrome; $R Y R 2$ for catecholaminergic polymorphic ventricular tachycardia; $M Y H 7$ and $M Y B P C 3$ for hypertrophic cardiomyopathy; $L M N A$ for dilated cardiomyopathy; and PKP2 and DSP for arrhythmogenic right ventricular dysplasia/ cardiomyopathy.

Results: The study included 17 male and 11 female patients; their mean age at diagnosis was 39 years (range, 1-80 years). The major clinical presentations included syncope, palpitations, and abnormal electrocardiography findings. A family history was present in $13(46 \%)$ patients. There were 26 different heterozygous mutations detected, of which six were novel-two in SCN5A (NM_198056.2:c.429del and c.2024-11T>A), two in MYBPC3(NM_000256.3:c.906$22 \mathrm{G}>\mathrm{A}$ and c.2105_2106del), and two in LMNA (NM_170707.3:c.73C>A and c.1209_1213dup).

Conclusions: We characterised the genetic heterogeneity in channelopathies and cardiomyopathies among Hong Kong Chinese interpretation of genetic findings is difficult and requires expertise and experience. Caution regarding issues of non-penetrance, variable expressivity, phenotype-genotype correlation, susceptibility risk, and digenic inheritance is necessary for genetic counselling and cascade screening.
Hong Kong Med J 2018;24:340-9
DOI: $10.12809 / \mathrm{hkmj} 176870$

\begin{abstract}
2 SPL Chen, MB, BS, FHKCPath
${ }^{3}$ NS Mok, MB, BS, FHKCP

${ }^{2}$ WK Siu, MB, BS, PhD

${ }^{2}$ HHC Lee, MB, ChB, MSc

${ }^{2}$ CK Ching, MB, ChB, MSc

${ }^{3}$ PT Tsui, MB, BS, FHKCP

${ }^{4}$ NC Fong, MB, BS, FHKCPaed

2 YP Yuen, MB, ChB, FHKCPath

${ }^{2}$ WT Poon, MB, ChB, FHKCPath

${ }^{2}$ CY Law, MB, ChB, PhD

${ }^{2}$ YK Chong, MB, ChB, FHKCPath

${ }^{2}$ YW Chan, MD, FHKCPath

${ }^{5}$ TC Yung, MB, BS, FHKAM (Paediatrics)

${ }^{6}$ KYY Fan, MBChB (Dundee), FHKAM (Medicine)
\end{abstract}
${ }^{1} \mathrm{CM}$ Mak *, PhD, MD
${ }^{7}$ CW Lam, MB, ChB, PhD

1 Chemical Pathology Laboratory, Kowloon West Cluster Laboratory Genetic Service, Department of Pathology, Princess Margaret Hospital, Laichikok, Hong Kong

Department of Pathology, Princess Margaret Hospital, Laichikok, Hong Kong

Department of Medicine, Princess Margaret Hospital, Laichikok, Hong Kong

${ }^{4}$ Department of Paediatrics and Adolescent Medicine, Princess Margaret Hospital, Laichikok, Hong Kong

${ }^{5}$ Department of Paediatric Cardiology, Queen Mary Hospital, Pokfulam, Hong Kong

${ }^{6}$ Department of Cardiac Medicine, Grantham Hospital, Wong Chuk Hang, Hong Kong

Department of Pathology, The University of Hong Kong, Pokfulam, Hong Kong patients in a 10-year case series. Correct

* Corresponding author: makm@ha.org.hk

New knowledge added by this study

- We characterised the genetic heterogeneity in channelopathies and cardiomyopathies among Hong Kong Chinese patients and described 26 mutations with six novel variants.

- This is the first case series of cardiac genetics in Hong Kong.

Implications for clinical practice or policy

- This study provides genetic information for variant interpretation and insight into the clinical application of genetic testing for channelopathies and cardiomyopathies. 


\section{Introduction}

Cardiac genetics is evolving rapidly and many new insights have recently been achieved. Genetic causes are found in various potentially lethal channelopathies and cardiomyopathies including long and short QT syndrome (LQTS and SQTS), Brugada syndrome, catecholaminergic polymorphic ventricular tachycardia (CPVT), hypertrophic cardiomyopathy (HCM), dilated cardiomyopathy (DCM), arrhythmogenic right ventricular dysplasia/ cardiomyopathy (ARVD/C), Barth syndrome, and left ventricular non-compaction. ${ }^{1}$ Knowledge of genetics deepens the understanding of pathophysiology and remarkably changes the diagnosis, treatment, and genetic counselling for recurrence risk and family planning. This group is highly genetically heterogeneous (Table $1^{2}$ ).

The genetic basis of inherited cardiac diseases in the Hong Kong Chinese population is largely unknown. The Princess Margaret Hospital provides a comprehensive cardiac genetic service. We conducted this study to review the clinical and genetic findings of 28 unrelated positive cases encountered between January 2006 and December 2015.

\section{Methods}

Diagnosis of the cardiac conditions was based on clinical assessments by a cardiologist and practice guidelines. ${ }^{3-5}$ The patients were referred by cardiologists from various public hospitals for genetic analysis. Only patients with positive genetic findings are reported in this study. There were seven patients with LQTS, two with Brugada syndrome, two with CPVT, nine with HCM, four with DCM, and four with ARVD/C. Local ethics board approval was obtained. Peripheral blood samples were collected from the proband after informed consent was obtained. Genomic DNA was extracted using

\section{香港華籍患者的心臟離子通道病和心肌病基因 檢測的情況：一所分區實驗室的經驗}

麥苗、陳柏林、莫毅成、蕭慧君、李漢芝、程楚君、徐炳添、 方乃聰、袁月冰、潘永達、羅震耀、張耀君、陳恩和、

\section{翁德璋、范瑜茵、林青雲}

引言：遺傳性心臟離子通道病和心肌病可以致命，它們的臨床表現多 樣化, 具遺傳異質性, 並至少與90個基因有關。基因檢測可以提供準 確診斷, 指導治療, 並進行直系家屬的連帶基因篩檢。香港華籍人口 在這兩種病的基因變異分佈尚未清楚。本文描述2006年1月至2015年 12 月期間，接受基因檢查並呈陽性結果的 28 名患者; 他們並沒有直接 的血緣關係。

方法：通過Sanger測序, 替沒有血緣關係的 28 名心藏離子通道病或 心肌病患者進行以下基因檢查：長QT綜合徵的 $K C N Q 1$ KCNH2 $K C N E 1 、 K C N E 2$ 和 $S C N 5 A$ 基因；Brugada綜合症的 $S C N 5 A$ 基因； 兒茶酚胺敏感性多形性室速的 $R Y R 2$ 基因; 肥厚型心肌病的 $M Y H 7$ 和 $M Y B P C 3$ 基因 ; 擴張型心肌病的LMNA基因 ; 致心律失常性右室心肌 病的PKP 2 和 $D S P$ 基因。

結果：患者 17 男和 11 女的平均確診年齡為39歲（介乎1-80歲）。主 要臨床表現包括軍厥、心悸和心電圖異常。13名患者 $(46.4 \%)$ 有家 族史。基因檢測結果發現有 26 個不同的基因變異, 其中有6個屬新發 現：有兩個在SCN5A基因中（NM_198056.2：c.429del和c.2024$11 \mathrm{~T}>\mathrm{A}$ ），兩個在MYBPC3基因中（NM_000256.3：c.906-22G>A 和c.2105_2106del），另有兩個在 $L M N A$ 基因中（NM_170707.3： c.73C $>A$ 和c.1209_1213dup）。

結論：在這十年病例回顧中, 我們對心臟離子通道病和心肌病的香港 華籍患者進行了基因分析。要正確解讀遺傳學結果需具備專業知識和 經驗。在遺傳諮詢和直系家屬的連帶基因節檢中, 必須對非外顯性 變異表達性、表型-基因型相關性、易感性風險和二基因遺傳問題謹慎 解讀。

a QIAamp Blood Kit (Qiagen, Hilden, Germany). The coding exons and the flanking introns (10 bp) of each gene were amplified by polymerase chain reaction. The primer sequences and protocol are available on request. Sanger sequencing was

TABLE I. Common channelopathy- and cardiomyopathy-associated genes ${ }^{2}$

\begin{tabular}{|c|c|}
\hline Disorder & Gene \\
\hline Long and short QT syndrome & $\begin{array}{l}\text { At least } 12 \text { genes: KCNQ1, KCNH2, SCN5A, ANK2, AKAP9, CACNA1C, CAV3, KCNE1, KCNE2, KCNJ2, } \\
\text { SCN4B, and SNTA1 }\end{array}$ \\
\hline Brugada syndrome & $\begin{array}{l}\text { At least } 16 \text { genes: SCN5A, CACNA1C, CACNB2, CACNA2D1, GPD1L, HCN4, KCNE3, KCND3, KCNE1L } \\
\text { (KCNE5), KCNJ8, RANGNRF, SCN1B, SCN2B, SCN3B, SLMAP, and TRPM4 }\end{array}$ \\
\hline $\begin{array}{l}\text { Catecholaminergic polymorphic } \\
\text { ventricular tachycardia }\end{array}$ & 2 Genes: RYR2 and CASQ2 \\
\hline Hypertrophic cardiomyopathy & $\begin{array}{l}\text { At least } 24 \text { genes: ACTC1, ACTN2, CSRP3, FXN, GLA, JPH2, LAMP2, LDB3, MYBPC3, MYH6, MYH7, } \\
\text { MYL2, MYL3, MYOZ2, PLN, PRKAG2, RAF1, TCAP, TNNC1, TNNI3, TNNT2, TPM1, TTN, and VCL }\end{array}$ \\
\hline Dilated cardiomyopathy & $\begin{array}{l}\text { At least } 30 \text { genes: TTN4, LMNA, MYH7, MYH6, SCN5A, MYBPC3, TNNT2, BAG3, ANKRD1, RBM20, } \\
\text { TMPO, LDB3, TCAP, VCL, TPM1, TNNI3, TNNC1, ACTC1, ACTN2, CSRP3, DES, NEXN, PSEN1, PSEN2, } \\
\text { SGCD, EYA4, PLN, DSG2, DMD, and TAZ }\end{array}$ \\
\hline $\begin{array}{l}\text { Arrhythmogenic right ventricular } \\
\text { dysplasia/cardiomyopathy }\end{array}$ & At least 8 genes: $D S C 2, D S G 2, D S P, J U P, P K P 2, R Y R 2, T G F B 3$, and TMEM43 \\
\hline
\end{tabular}


performed in the following order and stopped once a positive finding was detected: KCNQ1, KCNH2, KCNE1, KCNE2, and SCN5A for LQTS; SCN5A for Brugada syndrome; RYR2 for CPVT; $M Y H 7$ and MYBPC3 for HCM; LMNA for DCM; and $P K P 2$ and DSP for ARVD/C. The order was based on prevalence according to the literature and local experience. All coding exons were amplified for each gene except selected exons $3,8,14,45,46,47,49$, $88,89,90,93,96,97,100,101$, and 103 for $R Y R 2 .^{6}$ The GenBank accession numbers are shown in Table 2 . The pathogenicity of novel missense variants was analysed by Alamut Visual (Interactive Biosoftware, Rouen, France) with Polymorphism Phenotyping v2 (PolyPhen-2), Sorting Intolerant from Tolerant (SIFT), MutationTaster, and Assessing Pathogenicity Probability in Arrhythmia by Integrating Statistical Evidence (APPRAISE, https://cardiodb.org/ APPRAISE/) and that of novel splicing variants by Splice Site Finder-like, MaxEntScan, NNSPLIC, GeneSplicer, and Human Splicing Finder, wherever appropriate. Splicing variants were considered to be damaging if there was a $>10 \%$ lower score when compared with the wild-type prediction. Allele frequencies among populations were referred to the Exome Aggregation Consortium (ExAC; http://exac. broadinstitute.org/).

\section{Results}

During the 10-year study period more than 90 patients with channelopathies or cardiomyopathies were referred for genetic analysis. Among them, 28 unrelated patients had positive genetic results, comprising 17 males and 11 females. Their mean age at diagnosis was 39 years (range, 1-80 years). The major clinical presentations included syncope, palpitations, and abnormal electrocardiography (ECG) findings. Four patients were asymptomatic and were diagnosed following an incidental abnormal finding related to other medical issues. A family history was present in only 13 (46\%) patients. All detected mutations were heterozygous, and 26 different heterozygous mutations were detected. These encompassed 11 missense, two nonsense, and five splicing mutations, as well as eight small insertions and deletions. There were six novel mutations-two in SCN5A (NM_198056.2:c.429del and c.2024$11 \mathrm{~T}>\mathrm{A}$ ), two in MYBPC3 (NM_000256.3:c.90622G $>\mathrm{A}$ and c.2105_2106del), and two in $L M N A$ (NM_170707.3:c.73C >A and c.1209_1213dup) [Table 3]. All were considered pathogenic or likely pathogenic according to the Practice Guidelines for the Evaluation of Pathogenicity and the Reporting of Sequence Variants in Clinical Molecular Genetics by the Association for Clinical Genetic Science.? Further clinical details and genotypes are shown in Table 3.

There were seven patients with LQTS, two with Brugada syndrome, two with CPVT, nine with HCM, four with DCM, and four with ARVD/C. Three patients with LQTS had mutations in KCNQ1 (cases 1-3) and four had mutations in $\mathrm{KCNH} 2$ (cases 4-7). Two patients (cases 8 and 9) with Brugada syndrome had mutations in $S C N 5 A$, including two novel mutations. Two patients (cases 10 and 11) with CPVT had mutations in RYR2. Four patients with HCM (cases 12-15) had MYH7 mutations and five (cases 16-20) had MYBPC3 mutations, including two novel mutations. Four patients with DCM (cases 21-24) had $L M N A$ mutations, including two novel mutations. Finally, three patients with ARVD/C had PKP2 mutations (cases 25-27) and one had a DSP mutation (case 28).

\section{Discussion}

This is the first report of a cardiac genetic case series among Hong Kong Chinese patients with channelopathies and cardiomyopathies.

TABLE 2. Targeted genes in Sanger sequencing analysis

\begin{tabular}{|c|c|c|}
\hline Gene & Encoding & GenBank accession numbers \\
\hline KCNQ1 & Potassium channel, voltage-gated, KQT-like subfamily, member 1 & NG_008935.1, NM_000218.2, NP_000209.2 \\
\hline KCNH2 & Potassium channel, voltage-gated, subfamily $\mathrm{H}$, member 2 & NG_008916.1, NM_000238.3, NP_000229.1 \\
\hline KCNE1 & Potassium channel, voltage-gated, IsK-related family, member 1 & NG_009091.1, NM_000219.4, NP_000210.2 \\
\hline KCNE2 & Potassium channel, voltage-gated, IsK-related family, member 2 & NG_008804.1, NM_172201.1, NP_751951.1 \\
\hline SCN5A & Sodium channel, voltage-gated, type $\mathrm{V}$, alpha subunit & NG_008934.1, NM_198056.2, NP_932173.1 \\
\hline RYR2 & Ryanodine receptor 2 & NG_008799.1, NM_001035.2, NP_001026.2 \\
\hline MYH7 & Myosin, heavy chain 7, cardiac muscle, beta (MIM *160760) & NG_007884.1, NM_000257.2, NP_000248.2 \\
\hline МYВРСЗ & Myosin-binding protein $\mathrm{C}$, cardiac & NG_007667.1, NM_000256.3, NP_000247.2 \\
\hline LMNA & Lamin A/C & NG_008692.2, NM_170707.3, NP_733821.1 \\
\hline PKP2 & Plakophilin 2 & NG_009000.1, NM_004572.3, NP_004563.2 \\
\hline$D S P$ & Desmoplakin (MIM *125647) & NG_008803.1, NM_004415.2, NP_004406.2 \\
\hline
\end{tabular}


TABLE 3. Clinical and genetic findings of 28 Chinese patients with channelopathies and cardiomyopathies

\begin{tabular}{|c|c|c|c|c|c|c|c|c|c|}
\hline $\begin{array}{l}\text { Case } \\
\text { No. }\end{array}$ & $\begin{array}{l}\text { Sex/ } \\
\text { age at } \\
\text { diagnosis } \\
\text { (years) }\end{array}$ & Clinical details & $\begin{array}{l}\text { Family } \\
\text { history }\end{array}$ & Gene & $\begin{array}{l}\text { Heterozygous } \\
\text { mutation detected }\end{array}$ & ExAC & $\begin{array}{l}\text { PolyPhen-2 } \\
\text { SIFT } \\
\text { MutationTaster } \\
\text { APPRAISE PrPP } \\
\text { APPRAISE PoPP }\end{array}$ & $\begin{array}{l}\text { HGMD } \\
\text { accession }\end{array}$ & ClinVar \\
\hline \multicolumn{10}{|l|}{ LQTS } \\
\hline 1. & $M / 8$ & $\begin{array}{l}\text { Recurrent syncope induced by exercise } \\
\text { and swimming. Long QTc } 535 \text { ms and } \\
\text { broad-based T wave. }\end{array}$ & $\mathrm{N}$ & KCNQ1 & $\begin{array}{l}\text { c. } 1018 T>C \\
\text { p.Phe340Leu }\end{array}$ & Absent & $\begin{array}{l}\text { PD } \\
\text { D } \\
\text { DC } \\
0.98 \\
0.999\end{array}$ & CM1511175 & No entry \\
\hline 2. & $\mathrm{M} / 9$ & $\begin{array}{l}\text { Presented with an episode of unprovoked } \\
\text { syncope. ECG showed long QTc at } \\
490 \mathrm{~ms} \text {. Started on beta-blocker and } \\
\text { remained asymptomatic. }\end{array}$ & $\mathrm{Y}$ & KCNQ1 & $\begin{array}{l}\text { c. } 1032 \mathrm{G}>\mathrm{A} \\
\text { p.Ala344= }\end{array}$ & Absent & Exon-skipping & CS992245 & Pathogenic \\
\hline 3. & $\mathrm{~F} / 45$ & $\begin{array}{l}\text { Asymptomatic. ECG showed long QTc at } \\
505 \text { ms. Followed up because of strong } \\
\text { family history of sudden nocturnal death } \\
\text { and prolonged QT interval. }\end{array}$ & $\mathrm{Y}$ & $K C N Q 1$ & $\begin{array}{l}\text { c. } 1096 \mathrm{C}>\mathrm{T} \\
\text { p.Arg366Trp }\end{array}$ & Absent & $\begin{array}{l}\text { PD } \\
\text { D } \\
\text { DC } \\
0.98 \\
0.93\end{array}$ & CM981130 & $\begin{array}{l}\text { Pathogenic/ } \\
\text { likely } \\
\text { pathogenic }\end{array}$ \\
\hline 4. & $\mathrm{M} / 59$ & $\begin{array}{l}\text { Symptomatic bradycardia with dizziness. } \\
\text { One episode of loss of consciousness } \\
\text { during sleep. Long QTc at } 600 \mathrm{~ms} \text {, with } \\
\text { documented Torsades de pointes. ICD } \\
\text { implanted }\end{array}$ & $\mathrm{Y}$ & $\mathrm{KCNH} 2$ & $\begin{array}{l}\text { c.211G }>T \\
\text { p.Gly71Trp }\end{array}$ & Absent & $\begin{array}{l}\text { PD } \\
D \\
\text { DC } \\
0.96 \\
0.995\end{array}$ & CM1511185 & No entry \\
\hline 5. & $F / 31$ & $\begin{array}{l}\text { Asymptomatic, long QTc at } 494 \mathrm{~ms} \text { and } \\
\text { on beta-blocker. One offspring with LQTS } \\
\text { died of pneumonia in } 2005 \text {. }\end{array}$ & $Y$ & KCNH2 & $\begin{array}{l}\text { c. } 1750 G>C \\
\text { p.Gly584Arg }\end{array}$ & Absent & $\begin{array}{l}\text { PD } \\
\text { D } \\
\text { DC } \\
0.96 \\
>0.999\end{array}$ & CM097452 & Pathogenic \\
\hline 6. & $M / 1$ & $\begin{array}{l}\text { Congenital muscular ventricular septal } \\
\text { defect. Found bradycardia and long } \\
\text { QTc at } 560 \text { ms during follow-up at } 1 \\
\text { month old. Frequent runs of ventricular } \\
\text { tachycardia, pulseless ventricular } \\
\text { tachycardia with Torsades de pointes. } \\
\text { Pacemaker implanted. }\end{array}$ & $\mathrm{N}$ & $\mathrm{KCNH} 2$ & $\begin{array}{l}\text { c. } 1883 G>T \\
\text { p.Gly628Val }\end{array}$ & Absent & $\begin{array}{l}\text { PD } \\
\text { D } \\
\text { DC } \\
0.96 \\
>0.999\end{array}$ & CM055315 & Pathogenic \\
\hline 7. & $\mathrm{M} / 19$ & $\begin{array}{l}\text { Found with severe nausea, flaccid limbs, } \\
\text { eyes staring upwards, and frothiness in }\end{array}$ & $\mathrm{N}$ & $\mathrm{KCNH} 2$ & $\begin{array}{l}\text { c.2892dup } \\
\text { p.Gly965Argfs }{ }^{*} 154\end{array}$ & Absent & Frameshift & Cl097572 & No entry \\
\hline
\end{tabular}

mouth in bed in the morning. Also had

bilateral blurring of vision. Prolonged QTC at $550 \mathrm{~ms}$. ICD implanted.

Brugada syndrome

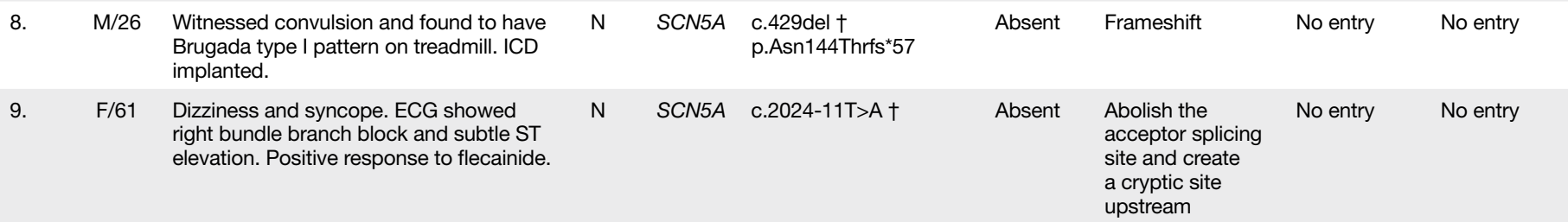

CPVT

\begin{tabular}{|c|c|c|c|c|c|c|c|c|c|}
\hline 10. & $F / 10$ & $\begin{array}{l}\text { Sudden cardiac arrest in school } \\
\text { playground. Ventricular tachycardia and } \\
\text { fibrillation in emergency department } \\
\text { requiring multiple defibrillations and } \\
\text { intensive care. Adrenaline infusion } \\
\text { test revealed bidirectional premature } \\
\text { ventricular beats and short runs of } \\
\text { polymorphic ventricular tachycardia. }\end{array}$ & $\mathrm{N}$ & $R Y R 2$ & $\begin{array}{l}\text { c. } 11836 G>A \\
\text { p.Gly3946Ser }\end{array}$ & Absent & $\begin{array}{l}\text { PD } \\
D \\
\text { DC }\end{array}$ & CM024349 & Pathogenic \\
\hline 11. & $\mathrm{M} / 7$ & $\begin{array}{l}\text { Exercise-induced recurrent loss of } \\
\text { consciousness. Echocardiogram showed } \\
\text { slightly thickened mitral valve and trivial }\end{array}$ & $\mathrm{N}$ & RYR2 & $\begin{array}{l}\text { c. } 14848 G>A \\
\text { p.Glu4950Lys }\end{array}$ & Absent & $\begin{array}{l}B \\
D \\
D C\end{array}$ & CM024354 & No entry \\
\hline
\end{tabular}
mitral regurgitation. Isoprenaline infusion test suggested CPVT. On beta-blocker.

Abbreviations: All = allele frequency in all populations; ARVD/C = arrhythmogenic right ventricular dysplasia/cardiomyopathy; $\mathrm{B}=$ benign predicted by PolyPhen-2; ClinVar = public archive of reports of the relationships among human variations and phenotypes (https://www.ncbi.nlm.nih.gov/clinvar/intro/); CPVT = catecholaminergic polymorphic ventricular tachycardia; $\mathrm{D}=$ damaging predicted by SIFT; DC = disease causing predicted by MutationTaster; DCM = dilated cardiomyopathy; EAS = allele frequency in East Asians; ECG = electrocardiography; ExAC = Exome Aggregation Consortium; HCM = hypertrophic cardiomyopathy; HGMD = Human Gene Mutation Database HGMD® Professional 20 17.2; ICD = implantable cardioverter defibrillator; LQTS = long QT syndrome; $\mathrm{MRI}=$ magnetic resonance imaging; $\mathrm{PD}=$ probably damaging predicted by PolyPhen-2; PoD = possibly damaging predicted by PolyPhen-2; PolyPhen-2 = polymorphism phenotyping V2; PoPP = posterior probability of pathogenicity predicted by APPRAISE which is only available for LQTS; PrPP = prior probability of pathogenicity predicted by APPRAISE, only available for LQTS; SIFT = sorting intolerant from tolerant

$\dagger$ Novel mutation 
TABLE 3. (cont'd)

\begin{tabular}{|c|c|c|c|c|c|c|c|c|c|}
\hline $\begin{array}{l}\text { Case } \\
\text { No. }\end{array}$ & $\begin{array}{l}\text { Sex/ } \\
\text { age at } \\
\text { diagnosis } \\
\text { (years) }\end{array}$ & Clinical details & $\begin{array}{l}\text { Family } \\
\text { history }\end{array}$ & Gene & $\begin{array}{l}\text { Heterozygous } \\
\text { mutation detected }\end{array}$ & ExAC & $\begin{array}{l}\text { PolyPhen-2 } \\
\text { SIFT } \\
\text { MutationTaster } \\
\text { APPRAISE PrPP } \\
\text { APPRAISE PoPP }\end{array}$ & $\begin{array}{c}\text { HGMD } \\
\text { accession }\end{array}$ & ClinVar \\
\hline \multicolumn{10}{|l|}{$\mathrm{HCM}$} \\
\hline 12. & $\mathrm{~F} / 60$ & $\begin{array}{l}\text { Detection of heart murmur during } \\
\text { routine check-up. Incidental finding of } \\
\text { obstructive HCM with mixed features of } \\
\text { apical and classic HCM. One sibling and } \\
\text { one offspring died of sudden cardiac } \\
\text { death in their } 30 \text { s. Her offspring had } \\
\text { HCM. }\end{array}$ & $\mathrm{Y}$ & MYH7 & $\begin{array}{l}\text { c. } 1273 G>A \\
\text { p.Gly425Arg }\end{array}$ & Absent & $\begin{array}{l}\mathrm{PD} \\
\mathrm{D} \\
\mathrm{DC}\end{array}$ & CM054791 & $\begin{array}{l}\text { Likely } \\
\text { pathogenic/ } \\
\text { uncertain } \\
\text { significance }\end{array}$ \\
\hline 13. & $\mathrm{M} / 57$ & $\begin{array}{l}\text { Exertional chest pain. Found to have } \\
\text { HCM. Refused to have prophylactic ICD } \\
\text { implantation. }\end{array}$ & $\mathrm{N}$ & MYH7 & $\begin{array}{l}\text { c. } 1987 C>T \\
\text { p.Arg663Cys }\end{array}$ & Absent & $\begin{array}{l}\mathrm{PD} \\
\mathrm{D} \\
\mathrm{DC}\end{array}$ & CM973126 & $\begin{array}{l}\text { Pathogenic/ } \\
\text { likely } \\
\text { pathogenic }\end{array}$ \\
\hline 14. & $\mathrm{M} / 59$ & $\begin{array}{l}\text { Irregular palpitations and hypertension. } \\
\text { Echocardiogram showed HCM. One } \\
\text { sibling had recurrent syncope and HCM } \\
\text { with ICD implantation. }\end{array}$ & Y & MYH7 & $\begin{array}{l}\text { c. } 1987 C>T \\
\text { p.Arg663Cys }\end{array}$ & Absent & $\begin{array}{l}\mathrm{PD} \\
\mathrm{D} \\
\mathrm{DC}\end{array}$ & CM973126 & $\begin{array}{l}\text { Pathogenic/ } \\
\text { likely } \\
\text { pathogenic }\end{array}$ \\
\hline 15. & $F / 48$ & $\begin{array}{l}\text { Episode of syncope. Found to have } \\
\text { HCM, with left ventricular dysfunction. } \\
\text { Progressive deterioration in exercise } \\
\text { tolerance. Heart transplantation done. } \\
\text { One parent died of cardiomyopathy and } \\
\text { sudden death in their 50s. One offspring } \\
\text { with incidental finding of heart murmur } \\
\text { and found to have HCM. }\end{array}$ & $\mathrm{Y}$ & MYH7 & $\begin{array}{l}\text { c. } 2155 \mathrm{C}>\mathrm{T} \\
\text { p.Arg719Trp }\end{array}$ & Absent & $\begin{array}{l}\text { PoD } \\
\text { D } \\
\text { DC }\end{array}$ & CM941086 & Pathogenic \\
\hline 16. & $\mathrm{M} / 52$ & $\begin{array}{l}\text { Recurrent syncope, loss of } \\
\text { consciousness, and episode of non- } \\
\text { sustained ventricular tachycardia. } \\
\text { Echocardiogram showed HCM. ICD } \\
\text { implanted. }\end{array}$ & $\mathrm{N}$ & МYВРC3 & c. $906-22 \mathrm{G}>\mathrm{A} \dagger$ & $\begin{array}{l}\text { All } \\
0.00089 \% \\
\text { EAS } 0 \%\end{array}$ & $\begin{array}{l}\text { Abolish the original } \\
\text { acceptor splice site } \\
\text { and create a cryptic } \\
\text { site }\end{array}$ & No entry & No entry \\
\hline 17. & $\mathrm{M} / 16$ & $\begin{array}{l}\text { Sudden collapse and ventricular } \\
\text { fibrillation, complicated by hypoxic brain } \\
\text { damage. Echocardiogram showed HCM. }\end{array}$ & $\mathrm{N}$ & МYBPC3 & c. $1223+1 \mathrm{G}>\mathrm{A}$ & Absent & $\begin{array}{l}\text { Abolish the original } \\
\text { donor splice site }\end{array}$ & No entry & Pathogenic \\
\hline 18. & $F / 43$ & $\begin{array}{l}\text { History of palpitations. Incidental finding } \\
\text { of pansystolic murmur at age } 36 \text { years. } \\
\text { Echocardiogram showed asymmetrical } \\
\text { intraventricular septal hypertrophy. Her } \\
\text { parents died in their } 40 \text { s of unknown } \\
\text { cause. }\end{array}$ & $\mathrm{N}$ & MYBPC3 & $\begin{array}{l}\text { c.2105_2106del † } \\
\text { p.Pro702Argfs*5 }\end{array}$ & Absent & Frameshift & No entry & No entry \\
\hline 19. & $F / 20$ & $\begin{array}{l}\text { Asymptomatic, incidental finding of } \\
\text { heart murmur referred from student } \\
\text { health service. Echocardiogram showed } \\
\text { thickened left ventricular septum. One } \\
\text { sibling also recently diagnosed with HCM } \\
\text { but echocardiograms of both parents } \\
\text { were normal. }\end{array}$ & $\mathrm{Y}$ & MYBPC3 & $\begin{array}{l}\text { c. } 2215 G>A \\
\text { p.Glu739Lys } \\
\text { c.3624del } \\
\text { p.Lys1209Serfs }{ }^{\star} 28\end{array}$ & $\begin{array}{l}\text { Absent } \\
\text { Absent }\end{array}$ & $\begin{array}{l}\text { PD } \\
\text { D } \\
\text { DC } \\
\text { Frameshift }\end{array}$ & $\begin{array}{l}\text { No entry } \\
\text { CD0910628 }\end{array}$ & $\begin{array}{l}\text { No entry } \\
\text { Pathogenic }\end{array}$ \\
\hline 20. & $M / 43$ & $\begin{array}{l}\text { Atypical chest pain and epigastric } \\
\text { discomfort. ECG showed typical features } \\
\text { of HCM. Severe septal hypertrophy. One } \\
\text { parent had HCM. }\end{array}$ & $\mathrm{Y}$ & MYBPC3 & $\begin{array}{l}\text { c.3624del } \\
\text { p.Lys1209Serfs } 28\end{array}$ & Absent & Frameshift & CD0910628 & Pathogenic \\
\hline \multicolumn{10}{|l|}{ DCM } \\
\hline 21. & $\mathrm{M} / 47$ & $\begin{array}{l}\text { History of craniopharyngioma and } \\
\text { found to have sick sinus syndrome and } \\
\text { third-degree heart block on preoperative } \\
\text { ECG. Echocardiography showed mildly } \\
\text { dilated right ventricle, mild tricuspid } \\
\text { regurgitation, mitral valve prolapse, } \\
\text { and trivial mitral regurgitation. Several } \\
\text { relatives had a history of atrioventricular } \\
\text { block. }\end{array}$ & $Y$ & LMNA & $\begin{array}{l}\text { c.48_51dup } \\
\text { p.Ser18Glnfs*24 }\end{array}$ & Absent & Frameshift & Cl117821 & No entry \\
\hline 22. & $\mathrm{~F} / 50$ & $\begin{array}{l}\text { Found unconscious at home. ECG } \\
\text { showed ventricular fibrillation. } \\
\text { Echocardiogram revealed DCM. Lower- } \\
\text { limb weakness and muscle wasting in } \\
\text { the past few years. One parent and one } \\
\text { sibling died of chronic heart failure in their } \\
30 \text { s or } 40 \text { s. The deceased sibling also } \\
\text { had heart block and muscular dystrophy. }\end{array}$ & $\mathrm{Y}$ & LMNA & $\begin{array}{l}\text { c. } 73 \mathrm{C}>\mathrm{A} \dagger \\
\text { p.Arg25Ser }\end{array}$ & Absent & $\begin{array}{l}\text { PD } \\
\text { D } \\
\text { DC }\end{array}$ & No entry & No entry \\
\hline 23. & $\mathrm{M} / 50$ & $\begin{array}{l}\text { Incidental finding of slow atrial flutter and } \\
\text { heart block. Echocardiogram showed } \\
\text { gross impairment of left ventricular } \\
\text { function and heart MRI revealed } \\
\text { anteroseptal and inferoseptal scars. }\end{array}$ & $\mathrm{N}$ & LMNA & $\begin{array}{l}\text { c.1209_1213dup † } \\
\text { p.His405Profs`77 }\end{array}$ & Absent & Frameshift & No entry & No entry \\
\hline
\end{tabular}


TABLE 3. (cont'd)

\begin{tabular}{|c|c|c|c|c|c|c|c|c|c|}
\hline $\begin{array}{l}\text { Case } \\
\text { No. }\end{array}$ & $\begin{array}{c}\text { Sex/ } \\
\text { age at } \\
\text { diagnosis } \\
\text { (years) }\end{array}$ & Clinical details & $\begin{array}{l}\text { Family } \\
\text { history }\end{array}$ & Gene & $\begin{array}{l}\text { Heterozygous } \\
\text { mutation detected }\end{array}$ & ExAC & $\begin{array}{l}\text { PolyPhen-2 } \\
\text { SIFT } \\
\text { MutationTaster } \\
\text { APPRAISE PrPP } \\
\text { APPRAISE PoPP }\end{array}$ & $\begin{array}{l}\text { HGMD } \\
\text { accession }\end{array}$ & ClinVar \\
\hline \multicolumn{10}{|c|}{ DCM (cont'd) } \\
\hline 24. & $\mathrm{~F} / 54$ & $\begin{array}{l}\text { Progressive limb weakness and } \\
\text { exertional dyspnoea. Mild elevation } \\
\text { of plasma creatine kinase at around } \\
1-2 \text { times the normal upper limit. } \\
\text { Echocardiogram showed dilated left } \\
\text { atrium, mildly reduced left ventricular } \\
\text { systolic function. Muscular dystrophy } \\
\text { with cardiomyopathy and atrioventricular } \\
\text { conduction disturbance. Put on } \\
\text { pacemaker. Two siblings had similar } \\
\text { conduction defects with permanent } \\
\text { pacing and muscle weakness. Their } \\
\text { muscle biopsies were unremarkable. }\end{array}$ & $\mathrm{Y}$ & LMNA & c. $1609-1 \mathrm{G}>\mathrm{A}$ & Absent & $\begin{array}{l}\text { Abolish original } \\
\text { acceptor splice site }\end{array}$ & CS1617015 & $\begin{array}{l}\text { Pathogenic/ } \\
\text { likely } \\
\text { pathogenic }\end{array}$ \\
\hline \multicolumn{10}{|c|}{ ARVD/C } \\
\hline 25. & M/62 & $\begin{array}{l}\text { Sudden onset of fast palpitations. ECG } \\
\text { at emergency department showed } \\
\text { ventricular tachycardia. ICD implanted. } \\
\text { Heart MRI showed right ventricular } \\
\text { dysplasia. History of anterior acute } \\
\text { myocardial infarction at age } 48 \text { years with } \\
\text { percutaneous coronary angioplasty done. }\end{array}$ & 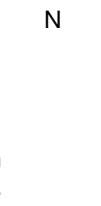 & PKP2 & $\begin{array}{l}\text { c. } 870 G>A \\
\text { p.Trp290* }\end{array}$ & Absent & Nonsense & CM1312955 & No entry \\
\hline 26. & $\mathrm{M} / 80$ & $\begin{array}{l}\text { Episodic palpitations with ECG showing } \\
\text { paroxysmal ventricular tachycardia. } \\
\text { Inducible ventricular tachycardia found } \\
\text { on electrophysiological study. ICD } \\
\text { implanted. }\end{array}$ & $\mathrm{N}$ & PKP2 & $\begin{array}{l}\text { c. } 1125 \text { 1132del } \\
\text { p.Phe376Alafs*8 }\end{array}$ & Absent & Frameshift & No entry & Pathogenic \\
\hline 27. & $\mathrm{M} / 49$ & $\begin{array}{l}\text { Recurrent palpitation attacks at } \\
\text { age } 39 \text { years. Sudden onset of } \\
\text { chest discomfort and palpitations } \\
\text { associated with dizziness and found } \\
\text { to have wide complex tachycardia. } \\
\text { Cardiac catheterisation revealed focal } \\
\text { aneurysm at right ventricular base. } \\
\text { Electrophysiological study revealed } \\
\text { inducible sustained ventricular } \\
\text { tachycardia with two different } \\
\text { morphologies. Heart MRI showed no } \\
\text { focal or diffuse right ventricular wall } \\
\text { thinning. }\end{array}$ & $\mathrm{N}$ & PKP2 & $\begin{array}{l}\text { c. } 1978 C>T \\
\text { p.Gln660* }\end{array}$ & $\begin{array}{l}\text { All } 0.00083 \% \\
\text { EAS } 0.012 \%\end{array}$ & Nonsense & CM100599 & No entry \\
\hline 28. & $F / 23$ & $\begin{array}{l}\text { Good past health. Found collapsed in } \\
\text { bathroom at home at night and death } \\
\text { confirmed at emergency department } \\
\text { arrival. Autopsy revealed mildly } \\
\text { dilated ventricles and mottling of } \\
\text { myocardium, with histological findings } \\
\text { of circumferential fibrofatty infiltration } \\
\text { of sub-epicardial region of left ventricle. } \\
\text { Right ventricle was essentially normal. }\end{array}$ & $\mathrm{N}$ & $D S P$ & $\begin{array}{l}\text { c.2131_2132delAG } \\
\text { p.Ser711Cysfs*4 }\end{array}$ & Absent & Frameshift & CD1410462 & $\begin{array}{l}\text { Pathogenic/ } \\
\text { likely } \\
\text { pathogenic }\end{array}$ \\
\hline
\end{tabular}

A total of 28 patients are reported, and 26 different mutations and six novel mutations have been identified. Wide genetic diversity is observed, with no common mutation found. Hereditary channelopathies and cardiomyopathies are mainly inherited in an autosomal dominant manner. Mutations can be either inherited or de novo. Risk to proband sibling(s) and first-degree relatives depends on the genetic status of the parents. Offspring of the proband have a $50 \%$ risk of inheriting the mutation. Siblings of the proband have the same risk if the mutation is transmitted from either parent. Patients carrying a mutation of these sudden arrhythmia death syndromes show incomplete penetrance. In general, a mutation carrier will show symptoms/ signs in $80 \%$ of those with CPVT, $20 \%$ to $50 \%$ of ARVD/C patients, $18 \%$ to $63 \%$ of LQTS patients, $80 \%$ to $94 \%$ of SQTS patients, and $80 \%$ of patients with Brugada syndrome who have abnormal ECG findings when challenged with a sodium channel blocker. ${ }^{8}$ No exact figure is available for HCM. The data could be more specific if a particular mutation was considered alongside clinical findings and family history. Pre-symptomatic testing of at-risk family members cannot be used to predict age of onset, severity, type of symptoms, or rate of progression. Detailed clinical, ECG, and genetic characterisation of affected and unaffected family members is helpful. 


\section{Long QT syndrome}

Long QT syndrome is genetically heterogeneous, with at least 12 genes involved. Mutations in the four genes, KCNQ1, KCNH2, KCNE1, and KCNE2, are detected in $46 \%, 38 \%, 2 \%$, and $1 \%$ of affected patients, respectively. ${ }^{8}$ A small proportion of patients (3\%) have double heterozygous mutations in more than one disease loci. ${ }^{9}$ Specific arrhythmogenic triggers are associated with a particular subtype, such as exertion, swimming, and near-drowning for LQT1; auditory triggers and cardiac events occurring in the postpartum period for LQT2; and cardiac events during sleep or at rest for LQT3. Three patients had KCNQ1 mutations. Case 1 had recurrent syncope induced by exercise and swimming, but genetic testing confirmed LQTS type 1. Other patients had no specific provoking factor. LQTS type 2 caused by $K C N H 2$ mutations accounts for about $38 \%$ of all LQTS. ${ }^{8}$ Four patients (cases 4-7) carried $\mathrm{KCNH} 2$ mutations and two (cases 4 and 6) presented with Torsades de pointes and one (case 7) had survived cardiac arrest requiring an implantable cardioverter defibrillator. Case 6 was the youngest patient, presenting at age 1 year. Genotype-guided treatment in LQTS is recommended and LQT1 responds best to beta-blockers. ${ }^{10,11}$

\section{Brugada syndrome}

Brugada syndrome is characterised by cardiac conduction abnormalities (ST-segment abnormalities in leads V1-V3 on ECG and a high risk for ventricular arrhythmias) that can result in sudden death. The Shanghai Score System has been recently published for the diagnosis of Brugada syndrome. ${ }^{12,13}$ The prevalence of Brugada syndrome or its characteristic ECG pattern is reportedly higher among Asians, such as Japanese (0.14\%-1.22\%). ${ }^{14-17}$

Brugada syndrome is genetically heterogeneous and can be attributed to defects in at least 23 genes at the time of reporting. ${ }^{8}$ Mutations in SCN5A are detected in $11 \%$ to $14 \%$ of affected individuals in Japan and $<10 \%$ in Taiwan where mutations in CACNA1C account for $1 \%$ to $7 \% .{ }^{18}$ Approximately $65 \%$ to $70 \%$ of patients remain genetically undiagnosed. Expressivity is variable and penetrance is incomplete and low.

Conventionally, Brugada syndrome has been described as a monogenic disease that has autosomal dominant inheritance with incomplete penetrance; it is caused by rare genetic variants with a large effect size. Most individuals diagnosed with Brugada syndrome have an affected parent. The proportion of cases caused by a de-novo mutation is approximately $1 \%$. Recent studies indicate that genetic inheritance is likely more complex, and models of an oligogenic disorder or susceptibility risk/genetic predisposition have been suggested. ${ }^{19-22}$

Among the two patients in this series, none had a positive family history. Symptoms were more non-specific, such as palpitation and syncope. It is noteworthy that convulsion can be a presentation of channelopathies (case 8). Clinical suspicion should be higher with more specific investigations, such as exercise-stress ECG and flecainide challenge tests, are required in order to reveal the real culprit. Sudden cardiac death can be the first presenting symptom in Brugada syndrome.

Two novel mutations are described in SCN5A: c.429del and c.2024-11T>A. The former is predicted to cause a frameshift and premature protein truncation. The latter is predicted to abolish the acceptor splice site and create a cryptic site upstream. At the time of reporting, both are absent from controls in the Exome Sequencing Project, 1000 Genomes Project, and ExAC. SCN5A mutations can cause either LQTS or Brugada syndrome.

\section{Catecholaminergic polymorphic ventricular tachycardia}

Catecholaminergic polymorphic ventricular tachycardia can present with syncope and sudden death during physical exertion or emotion, due to catecholamine-induced bidirectional ventricular tachycardia, polymorphic ventricular tachycardia or ventricular fibrillation. The reported mean age of onset is between 7 and 12 years. ${ }^{8}$ Exercise stress testing or an adrenaline provocation test may induce ventricular arrhythmia and enable a clinical diagnosis. About half of these cases are related to a dominantly inherited $R Y R 2$ gene mutation, with a small proportion (1\%-2\%) related to recessively inherited CASQ2 gene mutations. RYR2 is a large gene with 105 exons. Tier testing has been proposed by Medeiros-Domingo et al. ${ }^{6}$ First-tier $R Y R 2$ genetic testing of the 16 selected exons allows identification of about $65 \%$ of CPVT cases. There were two paediatric CPVT patients (cases 10 and 11) in our series, with two known disease-causing mutations detected, namely NM_001035.2(RYR2):c.11836G $>$ A (p.Gly3946Ser $)^{23-26}$ and c.14848G $>\mathrm{A}$ (p.Glu4950Lys). ${ }^{23,24}$ Both mutations were detected in first-tier screening.

\section{Hypertrophic cardiomyopathy}

Hypertrophic cardiomyopathy is the most prevalent hereditary cardiac disease, causing about one third of sudden cardiac deaths in young athletes. Its prevalence in China is approximately 1 in $1250 .{ }^{27}$ The clinical manifestations are markedly variable, ranging from asymptomatic to sudden cardiac death. Genetic testing provides an accurate diagnosis in the probands and enables screening of asymptomatic family members. Although the genetic background of $\mathrm{HCM}$ is heterogeneous, involving at least 30 genes, $M Y H 7$ and $M Y B P C 3$ are the most common and each accounts for approximately $40 \%{ }^{8}$ 
Nine patients with HCM are reported here: four had known $M Y H 7$ mutations and five had $M Y B P C 3$ mutations, including two novel mutations. NM_000256.3:c.906-22G>A was detected in case 16 and was a novel variant. Neither population frequency nor known pathogenicity have been reported. In-silico analysis showed creation of a novel acceptor site and insertion of 20 nucleotides into exon 10. This conceivably would lead to a frameshift and premature protein termination. Exon 10 of $M Y B P C 3$ is a microexon in which the stability of its original splicing site is easily disrupted by intronic variants. A similar mutation has been reported as c. $906-36 \mathrm{G}>\mathrm{A} .{ }^{28}$ Nonetheless, cDNA analysis was not performed. NM_000256.3(MYBPC3):c.1223+1G >A at the critical canonical +1 splice site is also novel. In addition, other known disease-causing splicing mutations affecting the same nucleotide have been reported. ${ }^{26,29,30}$ Case 19 had two variants detected in MYBPC3 (c.2215G>A and c.3624del). The small deletion c.3624del is a mutation known to cause $\mathrm{HCM}$ in the Chinese population ${ }^{31}$ and predicted to cause a frameshift and premature termination of the protein. The missense variant c.2215G $>\mathrm{A}$ is as yet unreported and is predicted by in-silico analyses to cause an amino acid change from glutamate to lysine at codon 739 and probably damage. At the time of reporting, the variant is absent from controls in the Exome Sequencing Project, 1000 Genomes Project, and ExAC databases. This variant is considered to have uncertain significance. The mother of the patient in case 19 was available for testing. She was 48 years old at the time of genetic testing, asymptomatic, and heterozygous for c.3624del only. Hence, the two variants c.2215G>A and c.3624del of $M Y B P C 3$ were in-trans in the patient and elder brother of the patient in case 19. Both had a more severe form of HCM, with a younger onset.

\section{Dilated cardiomyopathy}

Familial DCM is a group of genetically heterogeneous disorders. Laminopathy can manifest as several allelic disorders affecting muscle, nerve, adipose, and vascular tissues; one of them is cardiomyopathy, dilated 1A. We identified four patients with DCM, two of whom also had proximal muscle weakness. Two novel mutations in $L M N A$ were detected (c.73C $>\mathrm{A}$ and c.1209_1213dup). NM_005572.3(LMNA):c.73C $>\mathrm{A}$ is a novel variant that is predicted to be deleterious by SIFT, probably causing damage according to PolyPhen-2 and diseasecausing according to MutationTaster. Other missense mutations have been reported in the same amino acid codon. ${ }^{32-34}$ NM_005572.3(LMNA):c.1609-1G>A is predicted to significantly affect splicing by insilico analysis. At the time of reporting, all variants are absent from controls in the Exome Sequencing Project, 1000 Genomes Project, and ExAC. In case
22 with NM_005572.3(LMNA):c.73C >A, one of the parents died of chronic heart failure in the fourth decade of life, and one sibling died of heart block and chronic heart failure with a diagnosis of muscular dystrophy at age 38 years. Nonetheless, there was no sample left for genotyping.

\section{Arrhythmogenic right ventricular dysplasia/ cardiomyopathy}

Arrhythmogenic right ventricular dysplasia/ cardiomyopathy is associated with fibrofatty replacement of cardiomyocytes, ventricular tachyarrhythmias, and sudden cardiac death. Although the right ventricle is primarily affected in this condition, left-dominant arrhythmogenic cardiomyopathy has also been described, and mutations have been identified in DSP as well as in other genes. ${ }^{35}$ Four patients are reported here, with three having mutations in PKP2 and one in DSP. Interestingly, the patient in case 26 presented at age 80 years with episodic palpitations. His ECG results showed paroxysmal ventricular tachycardia. He had a deletion in PKP2, c.1125_1132del (p.Phe376Alafs"8), resulting in a truncated incomplete protein product. Age of onset in patients with PKP2 mutations is older than that of the patient with DSP mutation. The latter patient (case 28) died at age 23 years, with sudden collapse as the first presentation.

Primary arrhythmogenic disorders including LQTS/SQTS, CPVT, Brugada syndrome, and cardiomyopathies account for about one third of sudden cardiac deaths in the young. ${ }^{36}$ Identification of a pathogenic variant can solve the diagnostic mystery, provide relief to the family, and enable family screening and counselling for other at-risk family members. In some developed countries, molecular autopsy is an essential part of a formal forensic investigation in unexplained sudden death. ${ }^{37}$ We support the implementation of molecular autopsy in routine autopsy investigation of sudden cardiac death victims. Our group has conducted the first local prospective study to determine the prevalence and types of sudden arrhythmia death syndrome underlying sudden cardiac death among local young victims through clinical and molecular autopsy of sudden cardiac death victims and clinical and genetic evaluation of their first-degree relatives (http://www.sadshk.org/en/medical_research.php). Such data can serve as the groundwork for the feasibility of implementation of such investigations in Hong Kong.

Genetic tests for cardiac conditions can aid diagnosis and guide treatment. Nonetheless, there are limitations that complicate the translational use of genetic results in patient care, such as incomplete penetrance, variable expressivity, and findings of variants of uncertain significance. In addition, since the genetic heterogeneity is large among 
cardiomyopathies and channelopathies and more genes are yet to be discovered, a negative genetic finding does not necessarily exclude a genetic basis of disease in patients.

Major limitations of the current study include its small sample size, incomplete family data for cosegregation study, and lack of functional study of novel variants. We observed a lower rate of use of genetic tests in early years that might have been due to insufficient awareness among clinicians about the clinical usefulness of such tests for channelopathies and cardiomyopathies. Clinical indications published in an expert consensus statement on the state of genetic testing for channelopathies and cardiomyopathies from the Heart Rhythm Society and European Heart Rhythm Association provide a good reference to determine when a genetic test should be requested. ${ }^{5}$ In our hospital, referral information can be accessed on http://kwcpath. home/genetics/ and more information about genetic service provision in public hospitals is available in the Hong Kong Hospital Authority Genetic Test Formulary (http://gtf.home/). A comprehensive system of cardiac genetics service is required for an efficient referral system, resource funding, training, and appropriate long-term follow-up.

\section{Conclusions}

We present the phenotypic and genotypic characteristics of 28 unrelated Hong Kong Chinese patients diagnosed across a 10-year period. For each disease entity, it was beyond our reach in the past decade to exhaustively screen for all known genes. We therefore focus on the most common ones when investigating cardiac genetics. Even so, genetic analysis can provide an accurate diagnosis and is of utmost importance for the management of patients and their families. Non-penetrance, variable expressivity, phenotype-genotype correlation, susceptibility risk, and digenic inheritance have been reported. Genetic testing also allows for genetic counselling on the recurrence risk. Correct interpretation of genetic findings for careful genetic counselling requires professional expertise with relevant experience in both clinical medicine and molecular genetics. Next-generation sequencing will improve diagnostic performance in this genetically heterogeneous group of channelopathies and cardiomyopathies, and could become a mainstay diagnostic tool.

\section{Author contributions}

All authors have made substantial contributions to the concept or design of this study; acquisition of data; analysis or interpretation of data; drafting of the article; and critical revision for important intellectual content.

\section{Funding/support}

This research received no specific grant from any funding agency in the public, commercial, or not-for-profit sectors.

\section{Declaration}

All authors have no conflicts of interest to disclose. All authors had full access to the data, contributed to the study, approved the final version for publication, and take responsibility for its accuracy and integrity.

\section{Ethical approval}

Local ethical approval of this study was obtained (KW/EX/09155).

\section{References}

1. Herman A, Bennett MT, Chakrabarti S, Krahn AD. Life threatening causes of syncope: channelopathies and cardiomyopathies. Auton Neurosci 2014;184:53-9.

2. Ackerman MJ, Marcou CA, Tester DJ. Personalized medicine: genetic diagnosis for inherited cardiomyopathies/ channelopathies. Rev Esp Cardiol (Engl Ed) 2013;66:298307.

3. Priori SG, Wilde AA, Horie M, et al. HRS/EHRA/ APHRS expert consensus statement on the diagnosis and management of patients with inherited primary arrhythmia syndromes: document endorsed by HRS, EHRA, and APHRS in May 2013 and by ACCF, AHA, PACES, and AEPC in June 2013. Heart Rhythm 2013;10:1932-63.

4. Authors/Task Force members, Elliott PM, Anastasakis A, et al. 2014 ESC guidelines on diagnosis and management of hypertrophic cardiomyopathy: the task force for the diagnosis and management of hypertrophic cardiomyopathy of the European Society of Cardiology (ESC). Eur Heart J 2014;35:2733-79.

5. Ackerman MJ, Priori SG, Willems S, et al. HRS/EHRA expert consensus statement on the state of genetic testing for the channelopathies and cardiomyopathies: this document was developed as a partnership between the Heart Rhythm Society (HRS) and the European Heart Rhythm Association (EHRA). Europace 2011;13:1077-109.

6. Medeiros-Domingo A, Bhuiyan ZA, Tester DJ, et al. The RYR2-encoded ryanodine receptor/calcium release channel in patients diagnosed previously with either catecholaminergic polymorphic ventricular tachycardia or genotype negative, exercise-induced long QT syndrome: a comprehensive open reading frame mutational analysis. J Am Coll Cardiol 2009;54:2065-74.

7. Wallis Y, Payne S, McAnulty C, et al. Practice guidelines for the evaluation of pathogenicity and the reporting of sequence variants in clinical molecular genetics. Association for Clinical Genetic Science; 2013.

8. Adam MP, Ardinger HH, Pagon RA, Wallace SE, editors. GeneReviews [internet]. Seattle (WA): GeneReviews; 1993. Available from: https://www.ncbi.nlm.nih.gov/ books/NBK1116/. Accessed 19 Feb 2018.

9. Bokil NJ, Baisden JM, Radford DJ, Summers KM. Molecular genetics of long QT syndrome. Mol Genet Metab 2010;101:1-8.

10. Giudicessi JR, Ackerman MJ. Genotype- and phenotypeguided management of congenital long QT syndrome. 
Curr Probl Cardiol 2013;38:417-55.

11. Priori SG, Napolitano C, Schwartz PJ, et al. Association of long QT syndrome loci and cardiac events among patients treated with beta-blockers. JAMA 2004;292:1341-4.

12. Antzelevitch C, Yan GX, Ackerman MJ, et al. J-Wave syndromes expert consensus conference report: emerging concepts and gaps in knowledge. Europace 2017;19:665-94.

13. Antzelevitch C, Yan GX, Ackerman MJ, et al. J-Wave syndromes expert consensus conference report: emerging concepts and gaps in knowledge. J Arrhythm 2016;32:31539.

14. Furuhashi M, Uno K, Tsuchihashi K, et al. Prevalence of asymptomatic ST segment elevation in right precordial leads with right bundle branch block (Brugada-type ST shift) among the general Japanese population. Heart 2001;86:161-6.

15. Matsuo K, Akahoshi M, Nakashima E, et al. The prevalence, incidence and prognostic value of the Brugadatype electrocardiogram: a population-based study of four decades. J Am Coll Cardiol 2001;38:765-70.

16. Sakabe M, Fujiki A, Tani M, Nishida K, Mizumaki K, Inoue $\mathrm{H}$. Proportion and prognosis of healthy people with coved or saddle-back type ST segment elevation in the right precordial leads during 10 years follow-up. Eur Heart J 2003;24:1488-93.

17. Hiraoka M. Brugada syndrome in Japan. Circ J 2007;71 Suppl A:A61-8.

18. Juang JM, Tsai CT, Lin LY, et al. Unique clinical characteristics and SCN5A mutations in patients with Brugada syndrome in Taiwan. J Formos Med Assoc 2015;114:620-6.

19. Bezzina CR, Barc J, Mizusawa Y, et al. Common variants at SCN5A-SCN1OA and HEY2 are associated with Brugada syndrome, a rare disease with high risk of sudden cardiac death. Nat Genet 2013;45:1044-9.

20. Behr ER, Savio-Galimberti E, Barc J, et al. Role of common and rare variants in SCN1OA: results from the Brugada syndrome QRS locus gene discovery collaborative study. Cardiovasc Res 2015;106:520-9.

21. Gourraud JB, Barc J, Thollet A, et al. The Brugada syndrome: a rare arrhythmia disorder with complex inheritance. Front Cardiovasc Med 2016;3:9.

22. Juang JJ, Horie M. Genetics of Brugada syndrome. J Arrhythm 2016;32:418-25.

23. Priori SG, Napolitano C, Memmi M, et al. Clinical and molecular characterization of patients with catecholaminergic polymorphic ventricular tachycardia. Circulation 2002;106:69-74.

24. Jabbari J, Jabbari R, Nielsen MW, et al. New exome data question the pathogenicity of genetic variants previously associated with catecholaminergic polymorphic ventricular tachycardia. Circ Cardiovasc Genet 2013;6:481-9.

25. Kawamura M, Ohno S, Naiki N, et al. Genetic background of catecholaminergic polymorphic ventricular tachycardia in Japan. Circ J 2013;77:1705-13.

26. Xiong HY, Alipanahi B, Lee LJ, et al. RNA splicing. The human splicing code reveals new insights into the genetic determinants of disease. Science 2015;347:1254806.

27. Zou Y, Song L, Wang Z, et al. Prevalence of idiopathic hypertrophic cardiomyopathy in China: a populationbased echocardiographic analysis of 8080 adults. Am J Med 2004;116:14-8.

28. Frank-Hansen R, Page SP, Syrris P, McKenna WJ, Christiansen M, Andersen PS. Micro-exons of the cardiac myosin binding protein $\mathrm{C}$ gene: flanking introns contain a disproportionately large number of hypertrophic cardiomyopathy mutations. Eur J Hum Genet 2008;16:10629.

29. Millat G, Bouvagnet P, Chevalier P, et al. Prevalence and spectrum of mutations in a cohort of 192 unrelated patients with hypertrophic cardiomyopathy. Eur J Med Genet 2010;53:261-7.

30. Waldmuller S, Muller M, Rackebrandt K, et al. Array-based resequencing assay for mutations causing hypertrophic cardiomyopathy. Clin Chem 2008;54:682-7.

31. Liu X, Jiang T, Piao C, et al. Screening mutations of MYBPC3 in 114 unrelated patients with hypertrophic cardiomyopathy by targeted capture and next-generation sequencing. Sci Rep 2015;5:11411.

32. Narula N, Favalli V, Tarantino P, et al. Quantitative expression of the mutated lamin $\mathrm{A} / \mathrm{C}$ gene in patients with cardiolaminopathy. J Am Coll Cardiol 2012;60:1916-20.

33. Vytopil M, Benedetti S, Ricci E, et al. Mutation analysis of the lamin $\mathrm{A} / \mathrm{C}$ gene ( $L M N A)$ among patients with different cardiomuscular phenotypes. J Med Genet 2003;40:e132.

34. Yuan WL, Huang CY, Wang JF, et al. R25G mutation in exon 1 of $L M N A$ gene is associated with dilated cardiomyopathy and limb-girdle muscular dystrophy 1B. Chin Med J (Engl) 2009;122:2840-5.

35. Sen-Chowdhry S, Syrris P, Prasad SK, et al. Left-dominant arrhythmogenic cardiomyopathy: an under-recognized clinical entity. J Am Coll Cardiol 2008;52:2175-87.

36. Semsarian C, Hamilton RM. Key role of the molecular autopsy in sudden unexpected death. Heart Rhythm 2012;9:145-50.

37. Torkamani A, Muse ED, Spencer EG, et al. Molecular autopsy for sudden unexpected death. JAMA 2016;316:1492-4. 